\title{
Estados Unidos: ¡Hacer crecer el tenis, modificándolo!
}

\author{
Karl Davies
}

\author{
Asociación de Tenis de los Estados Unidos
}

RESUMEN

Desde el inicio de la campaña Play and Stay de la ITF en 2007 y desde el cambio de las Reglas de Tenis en 2012 para facilitar el entrenamiento y la competición en todo el mundo, la Asociación de Tenis de los Estados Unidos ha apoyado y promocionado estos cambios para mejorar el desarrollo y atraer y mantener a más jóvenes al juego de tenis. Para facilitar este desarrollo, desde el punto de vista de la programación, se empleó una visión más exhaustiva que incluyó las áreas siguientes: formación de entrenadores, entrenamiento, competición individual y por equipos, e instalaciones.
Palabras clave: tennis rules, projects, goals, education.

Recibido: 20 Dic 2016

Aceptado: 21 May 2017

Autor correspondiente: Karl

Davies Asociación de Tenis de

los Estados Unidos.

Correo electrónico:

karl.davies@usta.com

\section{INTRODUCCIÓN}

La misión de la Asociación de Tenis de los Estados Unidos, (USTA), es promover y desarrollar el deporte del tenis. Cuando nos encontramos ante este tipo de cambios, es decisión de la USTA tomar decisiones sobre el tenis, cumpliendo con la constitución, propósito y misión de la USTA (Hainline, 2012). Las Reglas de Tenis cambian muy excepcionalmente. Las Reglas de Tenis de la ITF cambiaron recién por quinta vez en la historia del juego, en 2012. Este cambio indica que las competiciones para menores de 10 años sólo se pueden jugar utilizando la pelota de etapa 3 (roja), etapa 2 (naranja) o etapa 1, (verde), y se podría utilizar una cancha más pequeña con métodos de puntaje de corta duración. La USTA decidió adoptar y fomentar un cambio de reglas que apoya los beneficios potenciales de la participación en el tenis, el desarrollo de los jugadores, y la promoción de la salud y del bienestar mediante el tenis (Schultz, 2012).

Los profesionales de la enseñanza y los entrenadores que aprenden a jugar al tenis usan estas pelotas más lentas y las raquetas más pequeñas desde hace años. En gran cantidad de casos, el material adaptado se usó como herramienta para que los tenistas iniciantes juveniles pudieran aprender las técnicas y las habilidades de golpeo básicas (Anderson, 2007).

El primer programa de material adaptado lanzado por la USTA fue Quickstart, un proyecto conjunto entre las divisiones de Desarrollo de jugadores y Comunidad de tenis. Los objetivos del proyecto eran:
1. Aumentar el número de jugadores de tenis iniciantes desde la edad de 5 años.

2. Aumentar la retención de jugadores de 5 a 10 años y más.

3. Mejorar el desarrollo técnico, táctico y físico de los jugadores de 5 a 10 años.

La USTA estudió otros programas de éxito en el mundo, y decidió que la forma de introducir el uso del material adaptado debía ser diferente. Había que investigar cada una de las oportunidades de juego disponibles para los niños. Estas oportunidades de juego incluían el juego informal, las prácticas por equipo y la competición individual y por equipos. El programa de juego modificado debería incluir a todos los niños que están comenzando o ya están en el tenis.

Durante el verano y el otoño de 2006, se iniciaron 26 programas piloto, involucrando a profesionales de la enseñanza, entrenadores de parques, clubes, campamentos, y colegios, probando seis especificaciones de edades, longitud y ancho de cancha, tamaño de la pelota, peso y bote, longitud de la raqueta, peso y tamaño de la empuñadura, altura de la red y sistemas de puntaje.

Una vez terminados los programas piloto, los proveedores debieron proporcionar la retroalimentación sobre su experiencia con el material modificado. Los resultados fueron muy positivos. La retroalimentación de los proveedores piloto mostró que los jugadores jugaban al tenis con poca instrucción formal, y competían y se divertían. Los niños tenían éxito jugando sin pasar por el tradicional modelo de aprendizaje de 
todos los golpes y de las tácticas antes de empezar a jugar (Anderson, 2007).

Además, se pidió retroalimentación a los padres de los jugadores participantes del programa piloto. Esta retroalimentación demostró que el juego les había parecido divertido y dinámico, y no reglamentado ni aburrido. Los entrenadores también expresaron una retroalimentación positiva, afirmando que sentían que los niños tenían más éxito con el juego modificado, y podían desarrollarse técnica y tácticamente (Anderson, 2007).

En el inicio oficial del programa Play and Stay, en el Abierto de los Estados Unidos de 2007, con Quickstart de la USTA, la retroalimentación expresó que este programa podría tener un enorme impacto en el tenis del país haciendo que los niños jueguen más pronto, mejorando más rápidamente tanto técnica como tácticamente, y permitiendo que estén más activos y energizados mientras juegan al tenis.

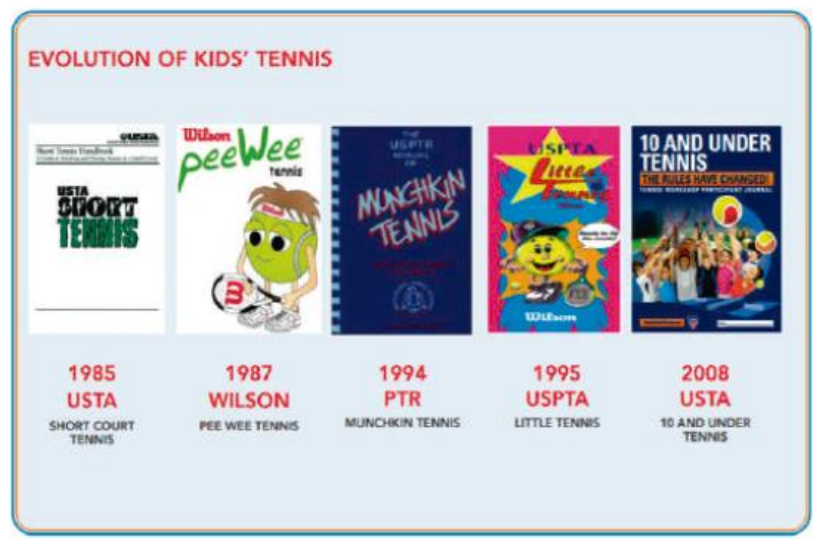

Figura 1. La evolución de los programas de tenis infantiles de la USTA.

En febrero de 2012, la USTA celebró el Simposio de Tenis Juvenil USTA en conjunto con el Instituto Nacional para la Salud y la Seguridad del Deporte de la Juventud (NYSHISI, por sus siglas en inglés). Debido a la falta de un plan científico para mejor introducir correctamente el programa a los menores de 10 años en los Estados Unidos, el propósito de este Simposio fue crear un programa para el desarrollo, entrenamiento y competición de jugadores menores de 10 años, y la transición a la categoría menores de 12 años. El propósito del NYSHISI era avanzar en su objetivo de demostrar el valor de la participación infantil en los deportes, también teniendo en cuenta la cantidad de riesgos de lesiones repetidas y burnout, en los casos de especialización temprana y forzada, y la profesionalización del deporte juvenil (Bergeron, 2012). El primer desafío incluido en la agenda del Simposio consistió en comprender que los niños no son pequeños adultos, por ende, hace falta un mayor estudio sobre sus características físicas, psicológicas, cognitivas, fisiológicas y sociales. Si no se tienen en cuenta estos factores, la estructura del programa de entrenamiento y competición podría perder su marca (Hainline,
2012). Otro tema tratado fue el desafío que implica, no solamente para el tenis, sino para otros deportes también, lograr cohesión entre el entrenamiento y la competición para los jóvenes atletas (Hainline, 2012). Otro interés particular, que también apareció en la agenda del Simposio, fue la necesidad de hablar sobre el desarrollo del carácter como componente clave para la formación de los jugadores. Otro punto comentado fueron los muchos mitos asociados con la el entrenamiento de la fuerza y el acondicionamiento de los niños. Si bien las ventajas que puede ganar un niño con el entrenamiento de la fuerza y el acondicionamiento tiene limitaciones, y existe un riesgo inherente de sobreentrenamiento, es evidente la importancia que tiene el desarrollo de las habilidades para la formación atlética de los niños (Schultz, 2012).

Desde el inicio de los conceptos Play and Stay, inicialmente Quickstart, y más recientemente, el tenis para menores de 10 años, y gracias al ímpetu del simposio celebrado en 2012, la USTA utiliza los siguientes programas para iniciar y mantener a más niños menores de 10 años en el deporte del tenis.

\section{Formación de entrenadores}

Comenzando con el Workshop para Entrenadores de Jugadores Aficionados, y luego con el Workshop para Entrenadores de Tenistas Juveniles, que comenzó en 2014, el propósito ha sido informar a la mayor cantidad posible de personas de los fundamentos del entrenamiento para menores de 10 años. El Seminario para Entrenadores de Tenistas Juveniles fue la primera de dos etapas de certificación en entrenamiento para menores de 10 años, en los Estados Unidos.

El Seminario incluye seis módulos de formación en línea y un Seminario presencial de 3,5 horas. Este último, de 3,5 horas, se ha cambiado por un curso en línea de 1,5 hora. Tras estos Seminarios introductorios para menores de 10 años, si el participante desea información extra, y una certificación, tomará entonces un curso, ya sea con la PTR (Professional Tennis Registry), o con la USPTA (Asociación de Tenis de los Estados Unidos), o con ambos. Estos talleres introductorios llegaron a más de 11.000 entrenadores y siguen aún involucrando a más entrenadores.

\section{Programa para escuelas}

El programa para Escuelas de la USTA es de alcance nacional y trata de facilitar la exposición al tenis en las escuelas, ofreciendo actividades de etapa pelota roja con los profesores de educación física, durante sus clases. Se proporciona entrenamiento y planes para clases por medio de un taller de 3 horas para los maestros.

Como una progresión, tras el programa de Educación física para tenis, se formaron clubes de tenis para niños, con el fin de darles la oportunidad de probar el tenis en un entorno seguro, de contención y extracurricular. 


\section{Tenis junior por equipos}

Tenis Junior por Equipos (JTT, sus siglas en inglés) es un programa nacional que intenta atraer a los jóvenes a competiciones por equipos. Además de ser divertido y una gran manera de hacer ejercicio, JTT es un medio competitivo, basado en los niveles, que fomenta el crecimiento individual, social y de las habilidades para la vida. Se compite en etapas de juego roja, naranja y verde, para los jugadores menores de 10 años. En algunas áreas, la etapa verde incluye a los jugadores menores de 12 años.

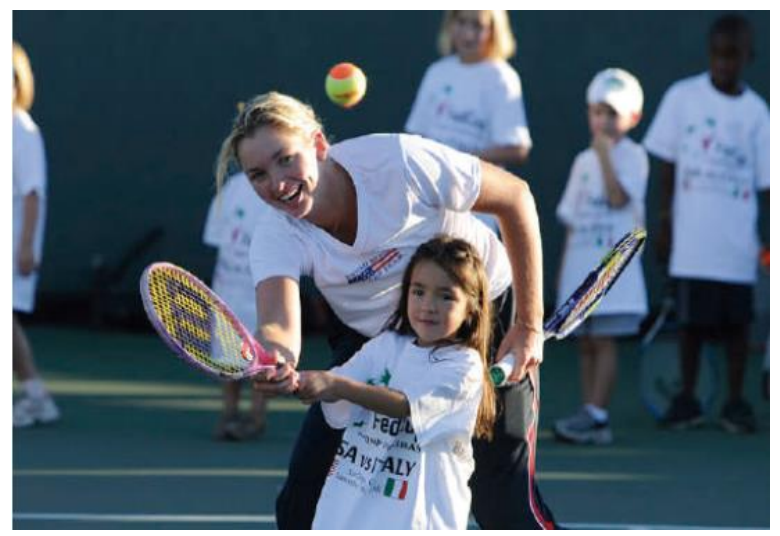

Torneos (progresión juvenil)

La progresión juvenil se diseñó para ayudar a los jugadores a mejorar sus fundamentos y aprovechar al máximo su experiencia en el tenis desde los niveles iniciales. El sistema de la progresión juvenil recompensa a los niños por la competición, y les inspira para que jueguen más. La misión es evidenciar cada nivel, compilando una colección de 20 estrellas y/ o trofeos virtuales por la participación. Cada sección de la USTA ofrecía una alternativa de cuatro opciones sobre cómo progresar de la etapa de pelota naranja a la verde y luego al juego con la pelota amarilla. En las etapas naranja y verde, los jugadores tienen la oportunidad de jugar competiciones individuales y por equipo, y les dan la posibilidad de ganar estrellas de participación, que hacen progresar a los jugadores entre las etapas. El propósito de la Progresión Juvenil era dirigirse más al entorno actual de los niños, por medio de un sistema lúdico.

\section{Campamentos de desarrollo temprano}

Los Campamentos de Desarrollo Temprano de etapas naranja y verde son el inicio del sendero Team USA diseñado para proporcionar oportunidades de entrenamiento extra a los tenistas menores de 10 años, en cada lugar de los Estados Unidos. Los Campamentos enfatizan las bases y el desarrollo atlético general. Los atletas invitados a estos campamentos tienen acceso a un aprendizaje divertido y formativo, mientras trabajan sus habilidades. Los jugadores seleccionados participarían también en los Eventos de Progresión Juvenil (torneos autorizados y JTT). Hay tres partes de Pelota Naranja y Verde, cada una incluye una presentación a los padres, un tema de habilidades mentales, y un formulario de retroalimentación jugador/ entrenador, incluido en el seguimiento del entrenador y la familia de cada participante.

\section{Instalaciones}

Para fomentar la mayor presencia del juego con material adaptado en distintas instalaciones en los EEUU, la USTA también ofrece becas para marcar líneas y lograr canchas de $36 / 60$ pies. Esta idea de las líneas no fue bien aceptada por los jugadores adultos quienes pensaban que perturbarían la visibilidad para su juego. La financiación variaría entre el 50$100 \%$ del costo de la instalación de las líneas. Hasta el presente, más de 20.000 canchas rojas y naranja se han beneficiado con esta beca.

\section{CONCLUSIÓN}

Como país de Grand Slam, el desarrollo del tenis, y la formación de la próxima generación de tenistas profesionales de altísimo nivel solo ocurrirá como consecuencia de atraer y mantener a más jóvenes en el juego de tenis. Se probó que el material adaptado logra el éxito del jugador, desde la primera vez en cancha, y le ayuda a aprender los fundamentos adecuados, técnica y tácticamente. La USTA, en su avance, continuará utilizando el concepto Play and Stay para mejorar y perfeccionar la programación para la juventud de los Estados Unidos.

\section{REFERENCIAS}

Anderson, K.A. (2007). Project 36/60 : une initiative de la Fédération américaine de tennis. ITF Coaching $\&$ Sport Science Review, 42, p. 17.

Bergerson, M. (2012). National Youth Sport Health and Safety Issues Perspectives. Communication présentée au symposium de I'USTA consacré à la pratique du tennis chez les jeunes, Tampa (Floride).

Hainline, B (2012). Positioning Youth Tennis For Success. White Plains, New York : USTA.

Schultz, S. (2012). The USTA 10 and Under Project: Broad Objectives. Communication présentée au symposium de I'USTA consacré à la pratique du tennis chez les jeunes, Tampa (Floride). 
CONTENIDO ITF ACADEMY RECOMENDADO (HAZ CLICKABAJO)

\section{ITF Academy}

Derechos de Autor (c) 2017 Karl Davies

\section{(c) (1)}

Este texto está protegido por una licencia CreativeCommons 4.0.

\begin{abstract}
Usted es libre para Compartir -copiar y redistribuir el material en cualquier medio o formato- y Adaptar el documento - remezclar, transformar y crear a partir del material- para cualquier propósito, , incluso para fines comerciales, siempre que cumpla la condición de:

Atribución: Usted debe dar crédito a la obra original de manera adecuada, proporcionar un enlace a la licencia, e indicar si se han realizado cambios. Puede hacerlo en cualquier forma razonable, pero no de forma tal que sugiera que tiene el apoyo del licenciante o lo recibe por el uso que hace de la obra.
\end{abstract}

Resumendelicencia - Textocompletodelalicencia 